\title{
A Comprehensive Survey of Location Based Routing in Vehicular Networks
}

\author{
Vikram Dhiman ${ }^{\text {a }}$ Ikjot Saini ${ }^{\text {b }}$, Prof Dr Manoj Kumar ${ }^{c}$ \\ ${ }^{a}$ PhD PTU, Punjab, 144001, India \\ ${ }^{b} \mathrm{PhD}$ University of Windsor, Canada, \\ ${ }^{c}$ Professor DAVIT, Jalandhar, 144001, India
}

\begin{abstract}
Vehicular network is the real time network formed by the highly dynamic nodes. This environment of VANET along with the fixed geographical mapping of roads slows down the delivery of the message in case of unicast and broadcast. When unicast is applied then the latency increases significantly as hop count increases. While broadcast make use of the network resources which degrades the performance by decreasing the efficiency of the network. However, multicast is proposed as the parameter to enhance the performance by introducing multicasting trees. This strategy is applied for maintaining the vehicular nodes in a multicast tree manner which will provide single path between two vehicles in the tree. Further, in case of the link failures, tree partitions and reconfiguration is needed this induces to have very low latency and reduces packet overhead. Various location based routing protocols are discussed which reveals different aspects and applications of variety of routing protocols relying on the location information.
\end{abstract}

Index Terms: LAR, Multicasting, VANET, Location based routing.

(C) 2017 Published by MECS Publisher. Selection and/or peer review under responsibility of the Research Association of Modern Education and Computer Science

\section{Introduction}

Vehicular networks are the adhoc environment having various vehicles and the roadside units. This network comprises no prior setup of infrastructure and the roadside units as well as the vehicles contact with each other in order to receive and send the warnings of the current traffic. Such messages transferring become significant in development of innovative safety technologies over different modes of transportation and traffic management applications. However, Intelligent Transportation System (ITS) makes safer and smarter use of the transportation by enabling users to be more informed and more coordinated. The technology is named as

* Corresponding author.

E-mail address: 
Wireless Access in Vehicular Environments signified as WAVE, is specially developed to adapt requirements of VANET and in order to support ITS and also known as IEEE 802.11p standard. PHY layer and IEEE 1609 for security and network management are the two layers of WAVE [1].

The vehicular environment has continuous movement of vehicles at different speeds that lead to unpredictable change in the topology of network. Since, there is limited time to exchange the information among vehicles or the roadside units. Various research projects on VANET are being carried out by researchers NoW (Network on Wheel) [4] which is carried out by a German Industry, FleetNet-Internet on the Road [3] which is focussed on the decentralization of the multihop adhoc networks and CarTalk [2] is the one that is developing the assistance to the drivers on road. These emphasis on deployment in the real world ITS goals for developing safety applications for the different modes of transportation. It can be done by providing the required information related to road, traffic and alerts at right time to drivers. Geocating routing protocols tends to utilize the location information obtained by Global Positioning System (GPS). The major geocasting protocols are Location Aided Routing [4] and Geographical Temporally Ordered Routing Algorithm [6] is modified from TORA, GeoGRID [7] is modified from GRID. Geocast Routing in Urban Vehicular Ad Hoc Networks [9] is especially for the city scenario where the traffic patterns are different from that of freeways and Distance Routing Effect Algorithm for Mobility, various mobility patterns are considered [8]. These protocols vary in the route discovery and route modification approach along with the movement of vehicles. By the use of information regarding location, the desired route is formed out of the reduced search space. Lesser number of route discovery messages are observes as the space for search decreases.

\subsection{Related Work}

Vehicular environment has a critical problem in designing the routing protocols due to the reason of having essential quality of the routing protocol that is the adaption of traffic patterns. Johnson and Maltz [11, 12] reveal the insufficiency of the conventional routing protocols for adhoc networks as the huge amount of wireless bandwidth may waste due to the routing related traffic.

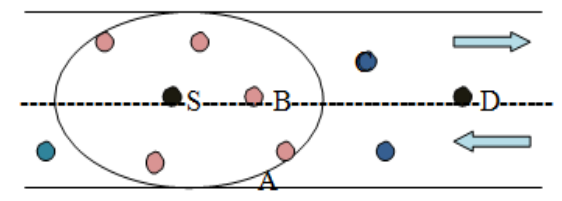

Fig.1. Forwarding Strategies

The categorization of vehicular networks has been done in three ways as cellular, adhoc and hybrid. First of all, cellular network, that deals with the local information and other infotainment facilities. In this case, vehicle and the roadside units communicate. This may provide a large number of applications but the drawback is the fixed infrastructure deployment. Further, it has been solved by the adhoc networks, where communication is among vehicles. Since the motion of the vehicles rapidly changes causing link failures, the problem exists for this paradigm as well. The optimized solution is the deployment of the roadside units along with the cellular networks which makes the hybrid communication link setup. This hybrid communication is significant in the position based routing as these are responsible for the maintaining the in the paper. Location information of the vehicles in its vicinity. The position based routing has following forwarding strategies:

- Greedy forwarding- Fig.1 depicts the scenario where in case of greedy forwarding, packet will be forwarded to ' $A$ ' by the source node ' $S$ ' as it is the closest node to the destination ' $D$ '.

- Improved greedy forwarding- The neighbour tables are consulted to compute the predicted positions for each neighbour. At time t1, source computes the prediction of neighbours that where they will be lying 
at $\mathrm{t} 2$ time instant. For this case, as 'B' overtakes 'A', therefore ' $\mathrm{S}$ ' selects ' $\mathrm{B}$ ' rather than ' $\mathrm{A}$ ' for next transmission node.

- Directional greedy forwarding- In this case, the next hop will be 'B' because it considers the direction of node. Selection is made on the basis of similar direction as of the destination.

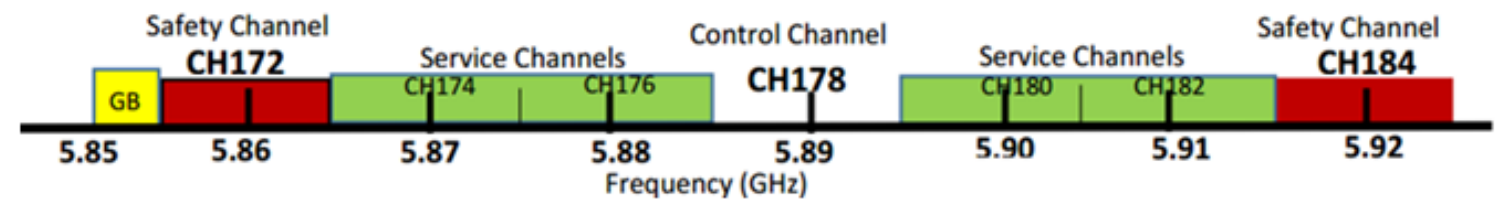

Fig.2. IEEE 802.11p Channel Frequency Band

- Improved greedy forwarding- The neighbour tables are consulted to compute the predicted positions for each neighbour. At time $\mathrm{t} 1$, source computes the prediction of neighbours that where they will be lying at 2 time instant. For this case, as ' $B$ ' overtakes ' $A$ ', therefore ' $S$ ' selects ' $B$ ' rather than ' $A$ ' for next transmission node.

- Directional greedy forwarding- In this case, the next hop will be ' $\mathrm{B}$ ' because it considers the direction of node. Selection is made on the basis of similar direction as of the destination.

- $\quad$ Predictive directional greedy forwarding- Two-hop neighbours are maintained in scheme. It consults its neighbour table before forwarding the packet and compute the prediction based on direction and speed of them. After that it selects a node out of its neighbours. According to Fig. 1, 'A' is selected because its moving in the direction of ' $\mathrm{D}$ ' and also it is the one-hop neighbour of ' $\mathrm{C}$ '.

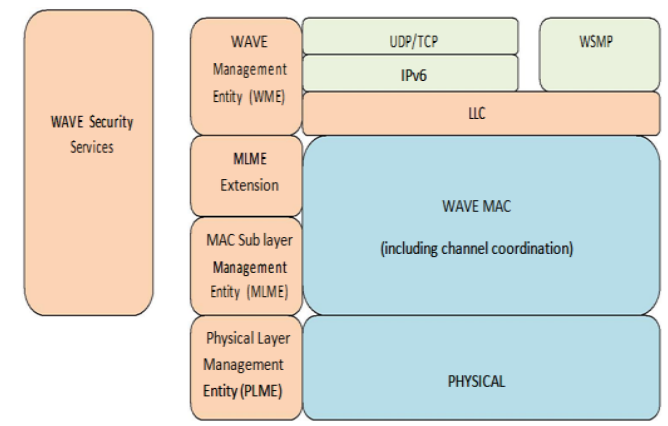

Fig.3. WAVE Protocol Stack

These forwarding strategies lead to the evolution of different routing protocols for various mobility patterns. DREAM is the protocol that maintains the location information in the routing tables of each vehicle. This protocol makes use of the Predictive Greedy Forwarding Strategy.

\section{IEEE 802.11p STANDARD (WAVE)}

The approved amendment to the standard IEEE802.11 leads to the evolution of the IEEE 802.11p in order to add Wireless Access in Vehicular Environment, WAVE protocol stack exhibit in Fig 3. Many applications are supported by this standard as it exchanges the data among the vehicles and the roadside units. The channel frequency band has been distributed as demonstrated in Fig. 2. The $5.9 \mathrm{GHz}$ frequency band with channel spacing of three different ranges enables it to be used in Europe and US. It uses DSRC (Dedicated Short Range 
Communication) technology. It provides V2V and V2I data exchange within $1 \mathrm{~km}$ range at transmission rate $3 \mathrm{Mbps}$ to $27 \mathrm{Mbps}$ when vehicle moving at speed of up to $260 \mathrm{~km}$ per hour. Two safety dedicated channels are there. The six channels are utilized for communication as bidirectional among different kinds of units. Physical layer (PHY) enables the exchange of frames [13]. PLCP is Physical Layer Convergence Protocol, the first layer that helps in communication and process of converging Packet Data Unit (PDU) into frame of OFDM.

\section{Location Based Routing Protocols}

Ko and Vaidya [5] propose LAR protocol which determine two of the zones, namely, request and expected. The rectangular area surrounds the sender as well as receiver. Expected zone covers the receiver where the possibility of it is high. The coverage is in the circular form as Fig. 4 demonstrates. The reduction in routing overheads is found by the decrease in the search area.

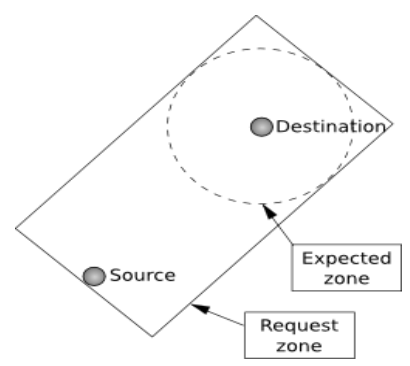

Fig.4. Location Aided Routing

Zaruba, Chaluvadi and Suleman [14] address Location Area Based Ad-Hoc Routing Protocol (LABAR), Fig.5 shows the area distribution. GPS enabled nodes are G-nodes which are interlinked. The dedicated nodes are responsible for location mapping to IP address, provide reliable exchange of information. It is the collaboration of two kinds of protocols, namely, reactive and proactive. As the virtual backbone disseminates the location information among G-nodes and also updates them and the directional routing takes place towards the destination's direction zone.

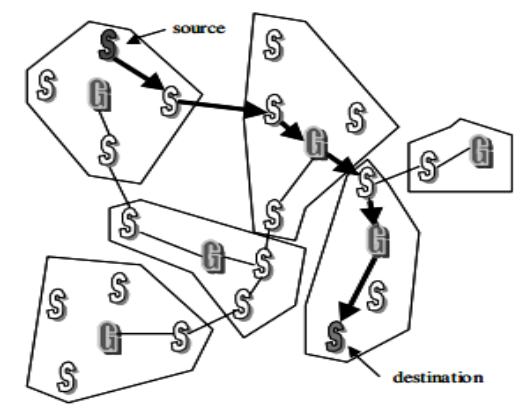

Fig.5. Location Area Based Ad-Hoc Routing Protocol (LABAR)

S. Basagi et al. [15] introduce Distance Routing Effect Algorithm for Mobility (DREAM) maintains the location routing tables of every node. Packet is forwarded on the basis of the knowledge of this location information. In order to have accurate location table, the periodic broadcast of control packet having the coordinates is carried out by each node. This concept introduces the accuracy and maintenance of the location information 
Karp and Kung [16] present Greedy Perimeter Stateless Routing (GPSR). It sends packet using the location information based on the distance. It utilizes the greedy forwarding strategy as shown in Fig.6. Sometimes there may be a case when the farther node from destination is best for the path. Such a scenario is applied upon by right hand rule for forwarding as soon as possible.

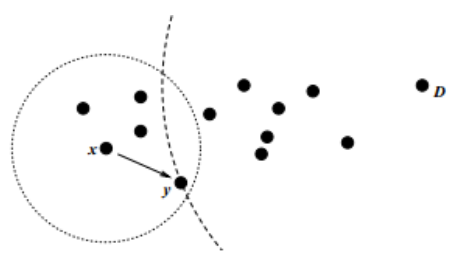

Fig.6. Greedy Perimeter Stateless Routing (GPSR)

Karim El Defrawy and Gene TsudikIn [19] propose the issues regarding suspicion regarding anonymous designing of routing framework (ALARM). A MANET map is formed by current locations of the nodes. On basis of this map, every node decides to which node it wants to establish the communication. It authenticates and also offers data integrity as well as intractability with anonymity which results in resistance to any of the attacker inside the network.

Tzay and Hsu[17] propose Location Aware Routing Protocol with Dynamic Adaptation of Request Zone for Mobile Ad hoc Networks (LARDAR). There are three advancements in this protocol. First of all, rectangular or triangular request zone determine coordinate information. This zone, shown in Fig.7, is smaller and thus provides smaller space for the route discovery. Additionally, for the adaption of the estimated precision of request zone, it comprises dynamic adaption technique for the triggering of the nodes lying immediate to destination. This results in more precise request zone. At last, for having a redo discovery of the route, a progressive search is carried out that uses angle basis increasing search whenever route discovery fails.

Haiying Shen and Lianyu Zhao[20] address Anonymous Location-based Efficient Routing protocol (ALERT). This provides low cost protection with high anonymity. As it divides the network field in certain zones dynamically, it selects random relay node that is an intermediate node used for transmission. Additionally, the identity of the initiator as well as receiver remains hidden among all other nodes providing anonymity protection. When compared with other anonymous routing protocols, this provides lower cost with high efficiency to GPSR.

Mohammad Al-Rabayah and Robert Malaney [21] present a protocol that collaborates two of the routing protocols. Reactive with the location based routing that gives efficient use of all available information regarding location. It makes spatial dependence of the various areas of the network at same epoch which can use different routing patterns. It enhances the scale at dramatic rate which can be calculated using routing control overhead.

Dan Luo and Jipeng Jhou [22] address a routing protocol with improved hybrid location approach which merges topology based routing with that of geographic. This reduces overall delay, problem of reactive routing. Further, average delay and packet delay rate also outperformed than that of pure reactive routing.

Lee, Yoo and Kim [23] present the improvement of the LAR protocol by considering node locations and the energy consumption. In order to have efficient routing, minimization of the unnecessary flooding of control messages is carried out. The prime focus is on the limited energy of mobile nodes and using it in optimized way. This proposal leads to the decrease the energy consumption with increase in average lifetime by 12 percent than that of LAR. 


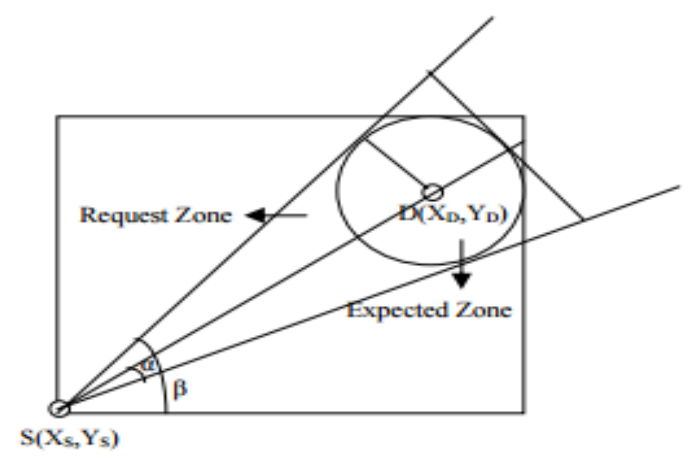

Fig.7. Location Aware Routing Protocol with Dynamic Adaptation of Request Zone for Mobile Ad hoc Networks (LARDAR)

Shanshan, Yanling, Yonghe, Mohan [24] propose Location Based Routing Scheme for Opportunistic Networks (LOOP) which forwards the packet to certain location rather than sending it to target node. The mobility traces are learnt by the patterns on which the nodes move. It helps in evaluating the performance of the protocol. It enables the message delivery at a quite high rate which abruptly decrease the load of network and occupancy of buffer of the nodes.

Mohammad A. Mikki [18] presented Energy Efficient Location Aided Routing Protocol (EELAR), extension to the LAR protocol. It focuses on the energy consumption by the mobile nodes. It restricts discovering area discovery to quite smaller area. It eventually causes reduction in the control packet overhead. A wireless reference base station has used along with the circular area with centre at the base station which further divide it into six areas distributed equally as shown in Fig. 8.The control packets are flooded to certain sub areas rather than flooding it to entire network in the route discovery phase. The mobile nodes' locations are kept with the base station in a position table.

Prakash Raj, Selva Kumar, Lekha [25] reveals Location Based Routing Protocol (LBRP) as the scheme operating without loop. It extracts dynamically topology to send packet to destination in a faster manner.

Kim, Young-Song, Hwang [26] address the data transmission with lesser consumption of the energy in stable manner. In this case, base station need to be aware of the nodes' location. The energy is balanced by all the nodes for entire lifetime of the network. It utilizes the cluster formation for the wider possible distributed environment.

Haidar Safa, Hassan Artail and Diana Tabet [26] present cluster based trust-aware routing protocol (CBTRP). The protection against the intermediary malicious nodes is the

Concern of this protocol. The organisation of the network is carried out in such a way that it elects the most trustworthy node among all as the cluster head which handles further routing activities. The trustworthiness is constantly ensured as soon as it is found to be malicious; dynamically updating is done for the packet path. It avoids malicious routes and makes the safer communication link setup.

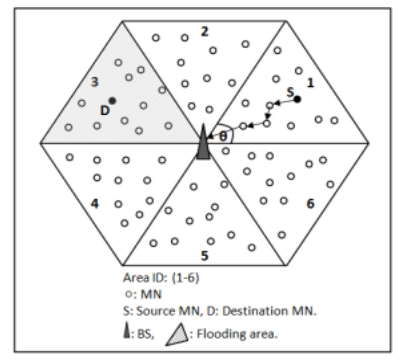

Fig.8. Energy Efficient Location Aided Routing Protocol (EELAR) 
Putthiphong Kirdpipat and Sakchai Thipchaksurat [27] propose Location-based Routing with Adaptive Request Zone (LoRAReZ) which reveals mobility impact. The scheme gives the adaptive area for the two zones, namely, request and expected. It relies on the source and destination distance.

Juanfei Shi and Kai Liu [28] gives power efficient location-based cooperative routing algorithm (PLCR) The analysis of the cooperative relay gives the successful packet reception probability which hike the overall routing power. The location information is utilized for taking the next node for transmission as well as cooperative node which comprising minimum power.

\section{Conclusions}

This paper reveals various categories of the routing protocols and the underlying forwarding mechanisms used in all the protocols. Several location based routing protocols has been discussed which differ in one way or another from each other. Route discovery and maintenance are the major concerns with the common purpose of maximization of throughput and reduction of the control packet overhead as well as overall delay and power consumption.

\section{References}

[1] Vehicular networks using the IEEE $802.11 \mathrm{p}$ standard: An experimental analysis by Fernando A. Teixeira,Vinicius F. e Silva, Jesse L. Leoni, Daniel F. Macedo, José M.S. Nogueira, Volume 1, Issue 2, April 2014, Pages 91-96

[2] http://www.cartalk2000.net/

[3] http://www.fleetnet.de/

[4] https://dsn.tm.kit.edu/english/projects_now-project.php

[5] Y. B. Ko and N. Vaidya, "Location-aided routing (LAR) in mobile ad hoc networks," in Proceedings of the ACM/IEEE International Conference on Mobile Computing and Networking (MOBICOM'98), 1998, pp. 66-75.

[6] Y. B. Ko and N. Vaidya, "Geocasting in Mobile Ad-hoc Networks: Location-Based Multicast Algorithms", In 2nd IEEE Workshop on Mobile Computing Systems and Applications, New Orleans, Louisiana, February 1999,pp. 101-110.

[7] Y. B. Ko and N. Vaidya, Geo TORA: A Protocol for Geo casting in Mobile Ad Hoc Networks. In: IEEE International Conference on Network Protocols, Osaka, Japan, 2000, pp.240-250.

[8] Stefano Basagni, Imrich Chlamtac, Violet R.Syrotiuk, and Barry A. Woodward, "A distance routing effect algorithm for mobility (DREAM)", In MobiCom'98:Proceedings of the 4th annual ACM/IEEE international conference on Mobile computing and networking (1998), pp. 76-84.

[9] Guoqing Zhang,, Wu. Chen, Zhong Xu,, Hong Liang, and Li Gao Dejun Mu, "Geocast Routing in Urban Vehicular Ad Hoc Networks", in R. Lee, and G.Hu, H. Miao, Eds. Computer and Information Science 2009, SCI 208, Springer-Verlag Berlin Heidelberg 2009,pp.23-31.

[10] E.Ahvar and M. Fathy, "Performance evaluation ofrouting protocols for high density ad hoc networks based on energy consumption by GlomoSim simulator" in the Proceedings Of World Academy Of Science, Engineering And Technology, Volume 23, August 2007. pp. 97-100.

[11] D.B. Johnson and D.A. Maltz, Dynamic Source Routing in Ad-Hoc Wireless Networks (Kluwer Academic, 1996)

[12] D.B. Johnson and D.A. Maltz, The Dynamic Source Routing protocol for mobile Ad-Hoc Wireless Networks (intenet draft), in: Mobile Adhoc Network (MANET) Working Group, IETF(1998).

[13] Kukshya, V. and H. Krishnan. Experimental measurements and modeling for vehicle-to-vehicle Dedicated Short Range Communication (DSRC) wireless channels. in Vehicular Technology Conference, 2006. VTC-2006 Fall. 2006 IEEE 64th. 2006. 
[14] Gergely V. Záruba, Vamsi K. Chaluvadi, and Azeem M. Suleman, "LABAR: Location Area Based Ad Hoc Routing for GPS-Scarce Wide-Area Ad Hoc Networks"IEEE;00, 2003, 7-7669955-1- 1889953;0-13.

[15] S. Basagni, I. Chlamtac, V.R. Syrotiuk, B.A. Woodward, "A distance routing effect algorithm for mobility (DREAM)" in: Proceedings of the ACM MOBICOM, pp. 76-84, 1998.

[16] B. Karp and H. Kung,. "GPSR: Greedy Perimeter Stateless Routing for Wireless Networks" in: Proceedings of ACM MobiCom, 2000, pp. 243-254

[17] Tzay-Farn Shih and Hsu-Chun Yen, "Location-aware routing protocol with dynamic adaptation of request zone for mobile ad hoc networks", Springer Wireless Network,14:321-333,DOI 10.1007/s11276-0069955-y, 2008.

[18] Mohammad A. Mikki, "Energy Efficient Location Aided Routing Protocol for Wireless MANETs" International Journal of computer science and Information Security, Vol. 4, No.1 \& 2, pp 331-337, 2009

[19] Karim El Defrawy, Member, and Gene Tsudik, Senior Member, "Privacy-Preserving Location-Based OnDemand Routing in MANETs" IEEE JOURNAL ON SELECTED AREAS IN COMMUNICATIONS, (29): 10,1926-1934, 2011

[20] Haiying Shen and Lianyu Zhao ALERT, "An Anonymous Location Based Efficient Routing Protocol in MANETs” IEEE TRANSACTIONS ON MOBILE COMPUTING, 2013, (12) 6:1079- 1093

[21] Mohammad Al-Rabayah, Robert Malaney, "A New Hybrid Location-based Ad Hoc Routing Protocol" IEEE Communications Society subject matter experts for publication, 978-1-4244-5638- 3/10, 2010

[22] Dan Lue \& Jipeng Jhou,."An Improved Hybrid Location Based Routing Protocol For Ad-H0c network" IEEE Network, 978-1-4244- 6252, Nov-2011

[23] Jangsu Lee, Seunghwan Y00, and Sungchun Kim, "Energy aware Routing in Location based Ad-hoc Networks" Proceedings of the 4th International Symposium on Communications, Control and Signal Processing, ISCCSP, 978-1-4244-6287-2, 3-5, Mar-2010

[24] Shanshan Lu, Yanliang Liu, Yonghe Liu and Mohan Kumar, "A Location Based Routing Scheme For Opportunistic Networks" IEEE Netwok, 978-1-4673-2433, 2012

[25] Dr. E. George Dharma Prakash, Raj, S. Selva Kumar and R. Lekha, "LBRP: GEOGRAPHIC ROUTING PROTOCOLS FOR MANETs" IEEE-International Conference on Recent Trends in Information Technology, ICRTIT, 2011, 978-1-4577-0590.

[26] Jin-I Kim, Jeong-Young Song, Yoon-cheol Hwang, "Location-based Routing Algorithm Using Clustering in the MANET" Future Generation Communication and Networking (FGCN), 2007, (2 ): 527 - 531,07695-3048-6.

[27] Haidar Safa ,Hassan Artail and Diana Tabet, “A cluster-based trustaware routing protocol for mobile ad hoc networks" Springer Science Business Media, LLC, 16:969-984, DOI 10.1007/s11276- 009-0182-1, 2010.

[28] Thipchaksurat Sakachai and Putthiphong Kirdpipat "Impact of Mobility on Location-based Routing with Adaptive Request Zone in Mobile Ad-hoc Networks” IEEE Network, 2012, 978-1-4673-2025.

\section{Authors'Profiles}

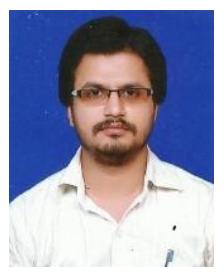

Er. Vikram Dhiman is currently pursuing PhD from PTU and has knowledge in multiple domain. He has published many research paper in the field of WSNs. He is also Red hat certified and successfully completing 10 years of experience in academic and Industry sector. His research interest in computer networks, Internet of things and cyber forensic. 


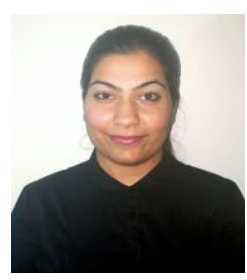

Ikjot Saini is currently working as graduate assistant and pursuing $\mathrm{PhD}$ in computer science from University of Windsor, Canada. She received her M.tech with specialization of computer networks and security from LPU, India. Her research interests includes computer networks, VANET, WSN and IT security. She is CISCO academy certified for routing and switching essentials. She has authored various technical research papers in the journals and conferences

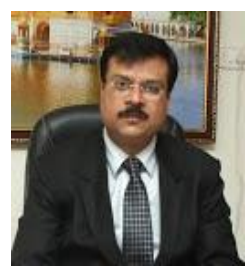

Professor Dr Manoj Kumar eminent researcher published 75 research paper in the field of optical and signal processing. He is an author of more than 8 books mainly in principle of communication and Analog communication system. He has supervised $15 \mathrm{M}$.Tech and 8 $\mathrm{PhD}$ thesis. His major project activities on optical soliton transmission system. He is currently working as a Principal, DAV Institute of Engg. and Technology.

How to cite this paper: Vikram Dhiman, Ikjot Saini, Manoj Kumar,"A Comprehensive Survey of Location Based Routing in Vehicular Networks", International Journal of Wireless and Microwave Technologies(IJWMT), Vol.7, No.1, pp.40-48, 2017.DOI: 10.5815/ijwmt.2017.01.05 